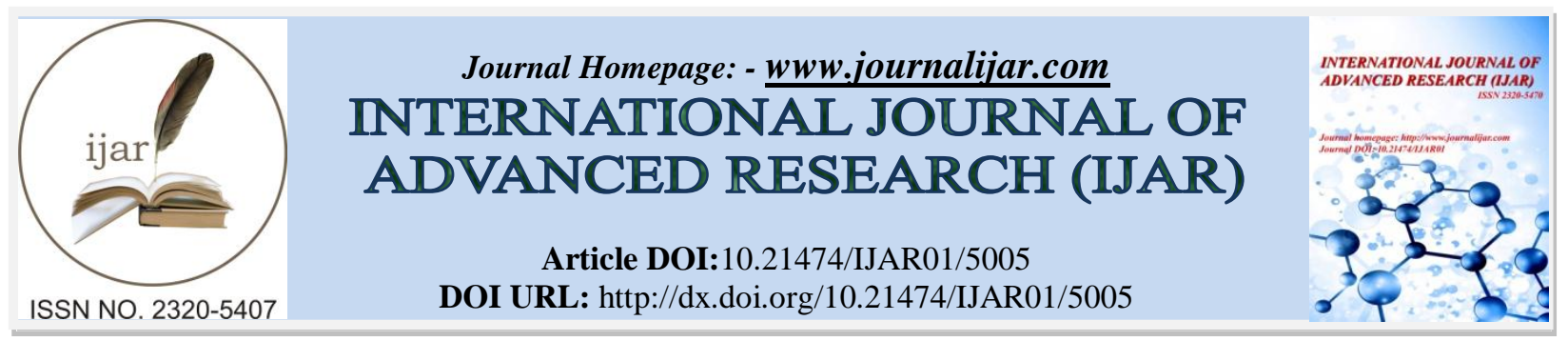

RESEARCH ARTICLE

\title{
A STUDY ON EFFECT OF ADDED NEUTRALISERS ON RENNET COAGULATION TIME (RCT) OF MILK.
}

\author{
Mr. Rajanna. M, Dr. Venkatesh M, Mr. Praveen. A.R And Mr. C Kempanna. \\ Dairy Science College Hebbal Bengalore, Karnataka, India.
}

\section{Manuscript Info \\ Manuscript History \\ Received: 29 May 2017 \\ Final Accepted: 31 June 2017 \\ Published: July 2017}

Key words:-

Rennet, Cheese, Paneer, Milk and

Flavour.

\section{Abstract}

The present paper attempts to study the effect of neutralizers on rennet coagulation time for milk. The findings reveals that, the rennet induced coagulation of milk is an essential and important in cheese manufacturing process, as it influences the quality and yield of cheese. Acidity favors coagulation of milk by rennet but alkalinity disfavors coagulation both acidity and $\mathrm{pH}$ were closely related to RCT for all types of milk. The calcium ions are added in the form of $\mathrm{CaCI}_{2}$ to speed up its activity, inhibitory substances like albumin and globulin retard clotting of milk and homogenization promotes clotting of milk. After neutralizations of sour milk it behaves mostly like normal milk as far as heat processing and other operations are concerned. However the quality of dairy products from neutralized milk is found to be very low. The cheese made from neutralized cow and mixed milk was observed to have yellowish color, loose body, more sticky texture where as cheese made from fresh buffalo milk has white in color, soft body, smooth texture and possessed pleasant flavor. Paneer prepared from neutralized milk showed soft body and smoky flavor. Smoky flavor increases with increase in the concentrations of neutralizers in milk. The yield of paneer is also reduced due to low recovery of total solids from neutralized milk.

Copy Right, IJAR, 2017,. All rights reserved.

\section{Introduction:-}

The milk with high - developed acidity is unsuitable for heat processing and as well as subsequent utilization in dairy products preparation. Thus it becomes imperative to neutralize high acid milk before it is heat processed. After neutralizations of sour milk it behaves mostly like normal milk as far as heat processing and other operations are concerned. However the quality of dairy product from neutralized milk is found to be very low (Marshall R.J Green M.L, 1980).

The Khoa made from neutralized cow and mixed milk was observed to have yellowish color, loose body, more sticky texture where as khoa made from fresh buffalo milk has white in color, soft body, smooth texture and possessed pleasant flavor ( Rajput, 1982). Paneer prepared from neutralized milk showed soft body and smoky flavor. Smoky flavor increases with increase in the concentrations of neutralizers in milk. The yield of paneer is also reduced due to low recovery of total solids from neutralized milk. 


\section{Mechanism of rennet coagulation of milk:-}

Milk clotting is a complex phenomenon involving both primary enzymatic phase and secondary non-enzymatic phase. In primary enzymatic phase rennin attacks on K-casein between phenylalanine-metheonine bond at 105-106 positions and liberates Para casein and glyco macro peptides. The coagulation of milk by rennet is initiated by the hydrolysis of a single peptide bond in K-casein, due to loss of highly negatively charged marcropeptide moiety of Kcasein (Dalgeish D.G 1984; Morrissey, 1989;

Mohamad K.S Fox .P.F (1983) the negative charge on casein micelles is reduced by rennet action and it has been suggested that the reduction in electrostatic repulsion may allow Vander Waals-attractive forces becomes dominant between the particles, so that aggregation occurs. Alternatively hydrophobic forces might be involved in aggregation and it has been suggested that micelles may interact through enzymitically-modified areas on their surfaces (Wilson and Wheelock, 1970).

In secondary non-enzymatic reaction, aggregation of altered casein micelles takes place as the stabilizing power of $\mathrm{K}$-casein destroyed by enzyme action. The caseinate micelles in milk become aggressively more susceptible to clotting in the presence of calcium ions. Clots will not firm in the absence of a calcium ion the secondary phase is particularly susceptible to variation in milk composition and to the presence of added salts (Hindle and Wheelock, 1970).

\section{Materials and Methods:-}

\section{Chemicals and Reagents:-}

The chemicals and reagents used were mainly of analytical grade. All the necessary reagents were prepared using distilled or doubled distilled water. The following chemicals were used in the investigation. Calcium chloride, Tartaric acid, Sodium hydroxide, Phenolphthalein indicator, Oxalic acid.

Milk:-

Composite cow and buffalo milk were collected from UAS Dairy farm and also some commercially available milk samples were also collected for the investigation.

\section{Equipments:-}

The following equipments were used for the investigation

1. High speed refrigerated centrifuge (Jouan BR4)

2. Systronic spectrophotometer

3. Cheese vat

4. Incubator

5. Water bath

6. $\mathrm{pH}$ meter (Eliko) and Conductivity meter (Eliko)

7. Starter cultures

Starter culture Materials

\section{Cultures:-}

Pure cultures of Streptococcus salivarius ssp, thermophilus, Lactococcus, delbreuki ssp, bulgaricus obtained from Dairy Microbiology lab. were used to prepare the fermented milks in the study.

\section{Rennet:-}

Rennet obtained from Meito sang go co. Ltd. (Japan) was used for the investigation.

\section{Neutralizers:-}

Chemical neutralizers such as sodium carbonate, sodium bicarbonate, and sodium hydroxide were used in the study.

\section{Methods:-}

Collection of milk sample:-

Milk samples collected from UAS Dairy Farm for the experiment. Sample is collected as per the standard procedures laid in ISO standards. 


\section{Rennet:-}

Rennet obtained from Meito sang go co. Ltd. (Japan) was used for the investigation.

\section{Neutralizers:-}

Chemical neutralizers such as sodium carbonate, sodium bicarbonate, and sodium hydroxide were used in the study.

\section{Determination of Rennet Coagulation Time (RCT):-}

Ten ml of milk samples were taken into screw cup test tubes and kept for 5 min in a thermostatically controlled water bath at $37^{\circ} \mathrm{C}$ to achieve renneting temperature. $0.5 \mathrm{ml}$ of rennet solution was added to each test tube, and inverting tubes mixed the contents, properly. The operation was carried out in thirty seconds. The time from the addition of rennet to the appearance and first visible flakes was noted down by stopwatch, duplicate determinations were carried out for all the samples (Anjum, 2000).

\section{Results and Discussions:-}

Quality of milk is paramount importance in the preparation of cheese. However preservation of quality of milk is becoming toughtest task due improper infracture facilities and collection of milk from long distance. During the transporation of milk from a far place the physic-chemical properties of milk are significantly affected, leading to undesirable behaviors in milk. Neutralization of sour milk could bring out changes in physico-chemical properties of milk such as $\mathrm{pH}$, acidity and salt balance etc. which have significant effect on physico-chemical properties of cheese milk such as thermal stability, rennet coagulability and buffering action of milk. This investigation was carried out to study the effect of different neutralizers such as sodium carbonate, sodium hydroxide and sodium bicarbonate on rennet coagulation time during cheese preparation and it is presented in table. 1.

Table 1:- Effect of added neutralizers on rennet coagulation time (min) of milk.

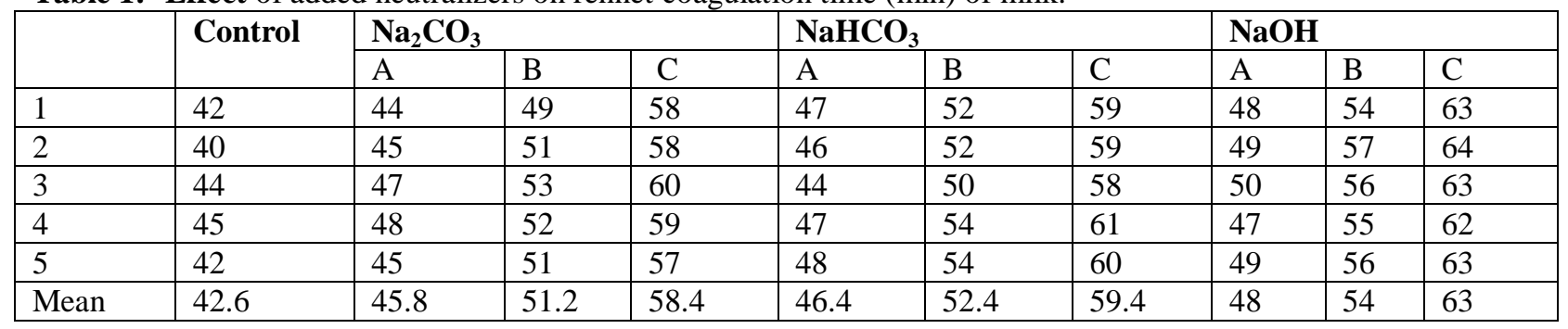

Table 2:- ANOVA.

\begin{tabular}{|l|l|l|l|}
\hline & CD & CV & F \\
\hline $\mathrm{Na}_{\mathbf{2}} \mathrm{CO}_{3}$ & 2.36 & 3.32 & 69.10 \\
\hline $\mathrm{NaHCO}_{3}$ & 2.68 & 3.81 & 63.56 \\
\hline $\mathbf{N a O H}$ & 2.047 & 2.86 & 104.2 \\
\hline
\end{tabular}

Control milk $0.15 \%$ lactic acid

A:-Milk neutralized from $0.20 \%$ lactic acid

B:-Milk neutralized from $0.25 \%$ lactic acid

C:-Milk neutralized from $0.30 \%$ lactic acid

Coagulation time for control milk samples was $42 \pm 1 \mathrm{~min}$. in the case of experimental samples, which were allowed to develop acidity levels of 0.2 . $0.25,0.30$, percent lactic acid and subsequently neutralized back to the normal acidity of control with different neutralizers were $45 \pm 1,51 \pm 1,58 \pm 1 \mathrm{~min}$, with respect to $\mathrm{Na} 2 \mathrm{CO} 3,46 \pm 1,52 \pm 1,59 \pm 1$ min, with respect to $\mathrm{NaHCO} 3$ and $48 \pm 1,54 \pm 1,63 \pm 1 \mathrm{~min}$, respectively with $\mathrm{NaOH}$ are presented in table.02. The maximum rennet coagulation time observed were for samples, which were neutralized from the acidity levels of 0.30 percent in all the three cases as shown in Table.02.

There was a significant effect of added neutralizers on RCT of milk. The RCT increases with increase in levels of neutralization with all the neutralizers. 


\section{Conclusion:-}

Rennet coagulation time is an important property, which has significance in the preparation of cheeses. RCT of milk decides the quality of final products. The RCT of milk is affected by various factors such as calcium ion concentration. Total solids content, acidity, temperature and $\mathrm{pH}$.

Milk with low or high RCT may not be suitable for cheese preparation as both of them produce undesirable product. Therefore, the investigation was conducted to ascertain the suitability of neutralized milk samples for the preparation of cheese. Significant increase in RCT of neutralized milk was noticed with increase in degree of neutralization of sour milk. The milk samples neutralized to the acidity of control from the acidity level of 0.30 and 0.35 per cent lactic acid showed significant increase in RCT.

Wilson G.A Wheelock (1972) also observed the increase in RCT of milk, which was acidified by the lactic acid bacteria and subsequently neutralized to the normal $\mathrm{pH}$. The increase in RCT in milk with added neutralizers may be attributed to the adsorption of cations on the casein micelles which mask the $\mathrm{K}$-casein micelles available to the enzyme hydrolysis and delays the RCT (Green and Marshall, 1976, 1980). The increase in RCT in neutralized milk also may be due to the disruption of micelles due to decrease in pH. Reddy.J.M and Kensella J.E (1990) reported the replacement of calcium by sodium during neutralization of milk by $\mathrm{Na}_{2} \mathrm{C} 03, \mathrm{NaHCO}_{3}$ and $\mathrm{NaOH}$. The calcium ions are important and are required for second phase of aggregation. Added neutralizers may compete for calcium ions and may lead to increase in RCT (Lucey, 1992). The effect of added neutralizers on RCT was studied. The results showed that there was a significant increases in RCT of neutralized milk sample and was proportional to the quantities of added neutralizers thus milk with added neutralizers may be unsuitable for cheese preparation.

\section{References:-}

1. Wilson, G.A. and Wheelock, J. V., 1972, Factors affecting the action of rennin heated milk. J. Dairy Res., 39 : 413-419.

2. Shalabi, S.I. and Weelock, J.V., 1976, Role of a-lactalbumin in the primary phase of rennet action on heated casein micelles. J. Dairy Res., 43 :331-335.

3. Olofclaesson, 1965, Studies on the variation of the rennin coagulation time of milk lart, brukshog, skolansannaler, 31: 238-327.

4. Reddy, J.M. and Kinsella, J.E., 1990, Interaction of (3-lactoglobulin with a- casein in micelles as assessed by chysmosin hydrolysis. Effect of temperature, heating time, (3-lactoglobulin, concentration and pH. J. Agric. Food Chem., 38 : 50-58.

5. Mohammad, K.S. and Fox, P.F., 1983, Influence of some polyvalent organic acids and salts on the colloidal stability of milk. J. Sci. Dairy Technol., $36: 112-117$.

6. Marshal], R.J. and Green, M.L., 1980, The effect of the chemical structure of additives on the coagulation of casein micelles suspensions by rennet. J. Dairy. Res., 47 : 359-369.

7. Lucey, J.A., 1992, Acid base buffering and rennet coagulation properties of milk systems. Ph.D. thesis National University of Ireland, Cork.

8. Dalgleish, D.G., 1984, Coagulation of renneted micelles dependence on temperature, calcium ion concentrations ionic strength. J. D. Res.,50:331-340. 